\title{
Cessation of Resuscitation in Pulseless Electrical Activity Out of Hospital Cardiac Arrest: A Mixed Methods Study of Senior Paramedic Decision Making
}

\author{
Alison Coppola ( $\square$ alison.coppola@swast.nhs.uk) \\ South Western Ambulance Service NHS Foundation Trust https://orcid.org/0000-0003-0135-3783 \\ Sarah Black \\ South western ambulance service NHS foundation trust \\ Ruth Endacott \\ University of Plymouth
}

\section{Original research}

Keywords: Paramedic, pulseless electrical activity, resuscitation

Posted Date: September 23rd, 2020

DOI: https://doi.org/10.21203/rs.3.rs-78106/v1

License: @ (1) This work is licensed under a Creative Commons Attribution 4.0 International License. Read Full License 


\section{Abstract}

Background

Pulseless electrical activity, a non-shockable cardiac arrest, is treated using advanced life support resuscitation. Typically, survival rates are poor and there is a paucity of evidenced-based guidelines for paramedics on when to cease resuscitation. This led to one UK Ambulance Service developing a local guideline to support senior paramedics when making cessation decisions. This study aimed to describe the patient characteristics, clinical features and system factors of pulseless electrical activity and explore the experiences of senior paramedics making autonomous cessation decisions.

Design and Methods

An explanatory sequential mixed method design conducted in a single UK Ambulance Service. Data was retrieved for all adult Out of Hospital Cardiac Arrest patients from $1^{\text {st }}$ December 2015 to $31^{\text {st }}$ December 2018. Cases subject to a coronial or police investigation were excluded. Senior paramedics ceased resuscitation for 50 PEA patients, a consecutive sample, which was reviewed in detail using descriptive statistics. Independent t-test and Chi Square examined associations between variables known to prognosticate survival. Interviews were conducted with six senior paramedics who remotely supported on-scene paramedics and findings were analysed using content framework analysis.

Results

Patient characteristics: Mean age 78 years, male $(n=30,60 \%)$, co-morbidities $(n=40,80 \%)$, witnessed collapse $(n=37,74 \%)$, bystander BLS $(n=30,60 \%)$. Clinical features: defibrillation $(n=22,44 \%), \operatorname{ROSC}(n=8,16 \%)$, heart rate $50(n=46,92 \%)$, mean $\mathrm{ETCO}_{2} 2.3 \mathrm{kPa}$. System factors: Advanced life support duration 59mins. A significant association between witnessed cardiac arrest and bystander basic life support was found $(95 \% \mathrm{p}=.00)$. Themes arising from interviews were defining resuscitation futility, the impact of ceasing resuscitation, conflicting views and clinical decision tools.

Conclusion

Senior paramedics interpreted and applied a multifactorial approach to ceasing resuscitation for patients with PEA. Patient characteristics, clinical features and system factors were balanced using clinical judgement, found to be vital to the decision-making process. This finding questions the appropriateness of paramedics making cessation decisions based on clinical features alone. As prognostic factors for survival were present, further investigation to identify the difference between an unsuccessful or futile resuscitation is required.

\section{Background}

Pulseless electrical activity (PEA) (previously known as electromechanical dissociation), a non-shockable cardiac arrest rhythm, is where the heart generates electrical activity, but muscle contractions are absent or too weak to generate a pulse.(1) PEA is the first documented rhythm in $31 \%$ of all out of hospital cardiac arrests in the UK, with $4.7 \%$ of patients surviving to hospital discharge.(2) Paramedics treat PEA with advanced life support (ALS), however, when treatment fails, patient survival is unlikely.(3) Given the low probability of survival, for some patients there may be a point where it is reasonable for the paramedic to cease resuscitation.

Prognosticating resuscitation outcomes was previously investigated to identify patients who benefit from continued resuscitation and those where resuscitation cannot succeed. $(4,5)$ Early recognition of cardiac arrest, basic resuscitation, defibrillation and post resuscitation care, (the chain of survival) when effective, can optimise patient survival.(3) In contrast, duration of cardiac arrest, co-morbidities, absence of a reversible cause and the return of spontaneous circulation (ROSC) should be considered when ceasing resuscitation.(3) Whilst senior paramedics may consider these patient characteristics and clinical features, UK guidelines state the evidence-base for PEA is limited. How senior paramedics decide to cease resuscitation for PEA has not previously been explored.(6)

\section{Methods}

\section{Study Aim}

This study aimed to describe PEA patients where resuscitation was ceased and explore the experiences of senior paramedics who provide remote telephone support to making autonomous cessation of resuscitation decisions. 
Objectives

1. To describe the patient characteristics, clinical features and system factors of PEA patients where resuscitation was ceased.

2. To examine the associations between variables known to optimise survival.

3. To explore the perceptions and experiences of senior paramedics autonomously making cessation of resuscitation decisions.

\section{Design and Setting}

This study employed an explanatory sequential mixed methods design. It was conducted in a single Ambulance Service NHS Foundation Trust providing emergency and urgent care services across an area of 10,000 square miles and a population of 5.5 million.

\section{Methods}

Cardiac Registry data was retrieved for all adult patients suffering an Out of Hospital Cardiac Arrest. Cases subject to a coronial or police investigation were excluded. A consecutive sample of 57 patients reported PEA as the first documented rhythm and senior paramedics ceased resuscitation in 50 patients which were reviewed in detail. Results from analysis of these data were used to inform semi-structured interviews. Interviews were conducted with senior paramedics who provide remote clinical support to on-scene paramedics.

\section{Patient and public involvement}

The Ambulance Service Patient and Public Involvement group, which included one cardiac arrest survivor supported this study. The term 'futile' was identified as meaning beyond all hope of patient recovery. The proposed research question and methodology was found appropriate. Group consensus was that paramedics should make cessation of resuscitation decisions based on individual circumstances. The group agreed they would not want resuscitation continued if their quality of life was expected to be poor.

Professional consultation with five research paramedics supported the study protocol. They felt paramedics should be empowered to cease resuscitation for PEA, supported by clear guidelines. They highlighted some paramedics may autonomously stop resuscitation and this would reduce the sample size. Bias may be introduced due to the quality of the retrospective data. A variety of interview platforms were proposed suitable for shift work commitments and anonymity assured due to the sensitive nature of resuscitation decisions.

\section{Data Collection and analysis}

\section{Patient data}

The Ambulance Service redacted identifiable patient information. Retrospective consent was not required as data was fully anonymised. Senior paramedics completed a cessation of resuscitation checklist for all PEA patients when resuscitation was ceased. All checklist data from December 2015 to December 2018 was screened against the inclusion criteria; adult cardiac arrest patients with PEA. Patients below 17 years, no PEA or those subject to coroner's court or a police investigation were excluded. Checklist data was cross referenced for accuracy for against clinical records, the computer aided dispatch system and the cardiac arrest registry. Checklist variables identified for analysis are presented in Table 1.

Table 1: Quantitative variables

\begin{tabular}{|lll|}
\hline \multicolumn{2}{|l|}{ Variables identified for analysis } & \\
\hline Patient Characteristics & System Factors & Clinical Features \\
\hline Age & Time of collapse & Adrenaline dose (mg) \\
\hline Gender & Time to basic resuscitation (response) & Progression to a shockable rhythm \\
\hline Arrest witnessed & Time to advanced resuscitation (response) & Defibrillation \\
\hline Bystander CPR & Duration of arrest & ETCO 2 prior to confirmation of death \\
\hline Co-morbidities & Duration of basic/advanced life support (BLS/ALS) & PEA heart rate \\
\hline Place of arrest & Total duration of out of hospital cardiac arrest & Return of spontaneous circulation \\
\hline Family wishes considered & & Rationale for terminating resuscitation \\
\hline
\end{tabular}


Descriptive statistics were applied to all variables identified in Table 1. We hypothesised there may be a difference in patients presenting with factors known to prognosticate survival (chain of survival: witnessed cardiac arrest, BLS and defibrillation) compared to those patients without. Independent t-test was used to examine duration of ALS between defibrillation/no defibrillation groups and between ROSC and non-ROSC groups. Chi Square examined association between witnessed cardiac arrest, BLS and defibrillation and ROSC. No effect size was required for this study and therefore no power calculation was applied. Statistical analysis was conducted using SPSS version 24.(7)

\section{Semi-structured interviews}

Six senior paramedics responsible for providing remote clinical support to on-scene paramedics were recruited as an expert sample during February and March 2019. The results from the quantitative phase informed the semi-structured questions for the qualitative phase. Interview questions were piloted with a critical care paramedic with one minor change required. Five interviews were by telephone, and one interview was conducted by email.

The study was underpinned by a pragmatic epistemology and critical realism ontology. Qualitative data was descriptive and applied content framework analysis. Existing theory was identified using deductive analysis and new theory identified using inductive analysis. Data was managed and constructed using NVIVO 11.4.2. (10)

Reflective validation was applied to the dialogue between the interviewer and interviewee to reduce bias and increase the trustworthiness and credibility of the data.(11) Reflexivity was supported using a diary to bracket assumptions and minimise the researchers influence during data collection and analysis. Data was cross coded by two independent researchers. The consolidated criteria for reporting qualitative research checklist was applied. (12)

\section{Ethics}

Institutional ethics approval was provided by the Trust (ref 17-018) and the University (reference 1718424).

\section{Results}

\section{Patient data results}

From the $1^{\text {st }}$ December 2015 to the $31^{\text {st }}$ December 2018, senior paramedics were contacted by the on-scene paramedic for clinical support for 57 PEA patients and resuscitation was ceased in 50.

Table 2 Patient Characteristics 


\begin{tabular}{|c|c|c|}
\hline & $\mathrm{N}(\%)$ & Median[IQR] \\
\hline \multicolumn{3}{|l|}{ Patient characteristics } \\
\hline Age, in years, median (IQR) & & 78 [69-84] \\
\hline Male & $30(60)$ & \\
\hline \multicolumn{3}{|l|}{ Location } \\
\hline Redidential address & $44(88)$ & \\
\hline Public place & $3(6)$ & \\
\hline Nursing/care residence & $3(6)$ & \\
\hline \multicolumn{3}{|l|}{ Witnessed } \\
\hline Yes & $37(74)$ & \\
\hline \multicolumn{3}{|l|}{ Bystander Resuscitation } \\
\hline Yes & $30(60)$ & \\
\hline \multicolumn{3}{|l|}{ Comorbidities reported ${ }^{+}$} \\
\hline Yes & $40(80)$ & \\
\hline \multicolumn{3}{|l|}{ Family wishes considered } \\
\hline Yes & $16(32)$ & \\
\hline$+51 \%$ of comorbidities wer & $8 \%$ resp & \\
\hline
\end{tabular}

Table 3 System factors and clinical features 
$\mathbf{N}(\%)$

\section{System factors*}

Duration hr:min:sec

No flow, median (IQR)

$50(100)$

$0: 07: 30(4.46-15.25)$

Duration of BLS median (IQR)

$26(52)$

$0: 07: 35$ (4.58-16.01)

Duration of lone ambulance BLS median (IQR)

$23(46)$

$0: 07: 32(5.31-15.9)$

Duration of ALS mean (SD)

$50(100)$

$0: 54: 46(0: 16: 59)$

Duration of resuscitation (BLS \& ALS) mean (SD)

$50(100)$

$0: 59: 32(0: 17: 39)$

Total duration of out of hospital cardiac arrest mean (SD)

$50(100)$

1:09:23 (0:19:53)

\section{Clinical features}

Defibrillation

ROSC achievied

End-tidal carbon dioxide (kilopascal, kPa)

Adrenaline Dose median (IQR)

PEA rate $<50 \mathrm{bpm}$

Notes

*No flow describes the time of collapse to onset of resuscitation. Lone ambulance responders were identified as community responders, advanced technicians, paramedics and duty officers, providing BLS only. ALS describes the time of ambulance arrival to confirmation of death, duration of out of hospital cardiac arrest was calculated as no flow BLS and ALS.

\$Missing data for 6 patients

PEA was the only reported rhythm for 18 (36\%) patients, 32 (64\%) reported a rhythm change and 20 (38\%) of these progressed to a shockable rhythm.

Associations between factors

Table 4 Association between factors

\begin{tabular}{|lll|}
\hline Factors & & \\
\hline Witnessed cardiac arrest & Bystander BLS & $\chi^{2}(n=50, \mathrm{df} 1)=p 0.000$ \\
\hline Witnessed cardiac arrest & Defibrillation & $\chi^{2}(n=50, \mathrm{df} 1)=p 0.856$ \\
\hline Bystander BLS & Defibrillation & $\chi^{2}(n=50, \mathrm{df} 1)=p 0.103$ \\
\hline Witnessed cardiac arrest & ROSC & $\chi^{2}(n=50, \mathrm{df} 1)=p 0.418$ \\
\hline Bystander BLS & ROSC & $\chi^{2}(n=50, \mathrm{df} 1)=p 0.875$ \\
\hline
\end{tabular}

Table 5 Difference in duration of ALS for groups with ROSC/no ROSC and receiving/not receiving defibrillation

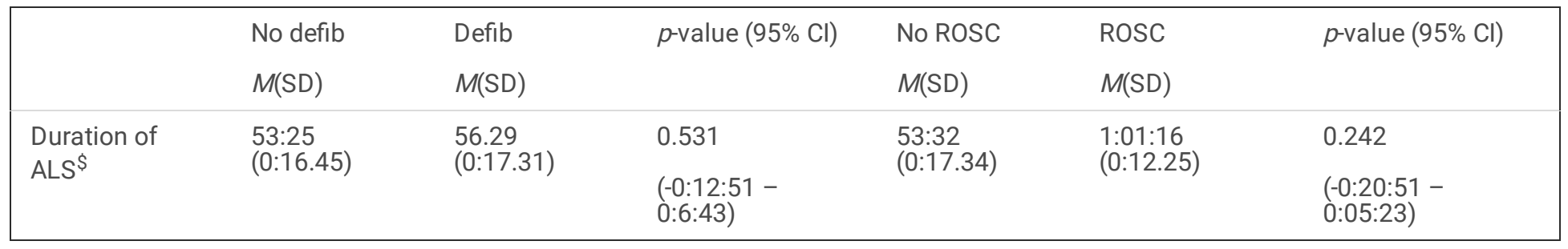


\$-test

No statistical significance between witnessed cardiac arrest or bystander resuscitation and defibrillation, witnessed or bystander resuscitation and ROSC were found. Nor were there any associations between age and co-morbidities and duration of resuscitation.

\section{Senior paramedic rationale for ceasing resuscitation}

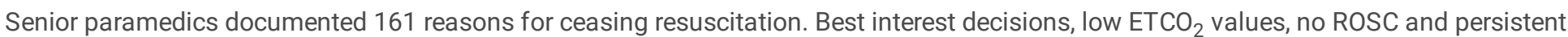
PEA were the most considered clinical features $(n=117,71.7 \%)$. Family wishes and progressive illness

were the most considered characteristics $(n=30,19.6 \%)$. Duration of resuscitation

and extended hospital transfer times were the most considered system factors ( $n=14,8.5 \%)$. These results identified a number of PEA patients that presented with factors known to prognosticate survival, witnessed cardiac arrest, bystander resuscitation, defibrillation, in addition to normal $\mathrm{ETCO}_{2}$ values, and ROSC, albeit intermittent. These results informed the semi structured interview questions to examine why resuscitation was ceased (Appendix 1).

Interview findings

The senior paramedics recruited were mixed gender, median age 40, ranging from 35-47 years. Average clinical experience was 14 years and 'on call' rota experience ranged from six months to nine years. Framework content analysis generated 349 codes, 12 sub-themes and four main themes: (1) Defining a futile resuscitation (2) The impact of ceasing resuscitation (3) perceived conflict between senior and onscene paramedics (4) supportive tools for ceasing resuscitation. Multifactorial decision-making informed by clinical judgement remained fluid throughout the data. An overview of themes and sub-themes with illustrative quotes is presented at Table 5.

Table 5: Themes, sub-themes and illustrative data excerpts 


\section{Theme \& sub- Data excerpt}

theme

Defining a futile resuscitation

Key features "things like age, rhythm, end tidal capnography, previous medical conditions and events preceding the event are probably some of the key factors l'd be thinking about (SP1)

A poor quality of life "quality of life I know there's lots of controversy about should you use that as an indicator but I find it useful to know in terms of if in the nicest possible way if the patients in a nursing home bedbound with severe dementia and comorbidities there's something about should we be resuscing anyway". (SP5)
Perceived natural end of life
"I may feel more confident to say actually if it was an older person or more of the elder category then to consider that that might be their natural end of life". (SP3)

The impact of ceasing resuscitation

Confidence

gained with experience and

exposure

Critical awareness

and self -

reflection
"I am confident in making a decision yes I've been making it for enough years so I feel confident in making it.... is it always clear cut? ... No". (SP5)

"They are difficult decisions to make and are often disruptive especially at night when you are woken up.... often I find it difficult to fall back to sleep as I reflect on my decision and feel a sense of loss for the family". (SP6)
The weight of responsibility when deciding futility "it did have its moments going about your daily business and you think bloody hell that is a bit hardcore so it's very easy writing out a word document on your form and filling it in but when you actually put someone's name in it and all their bits and pieces its quite oh actually someone just died and sometimes that was more stressful more poignant that being there". (SP4)

Perceived conflict between senior and on-scene paramedics

Conflict between senior and onscene paramedic "they are trying to sell me a situation to fulfil their own agenda so their selling me a patient that's profoundly unwell when there actually a survivable aspect to it because it is frustrating". (SP4)
Senior paramedic clinical

knowledge,

experience and

accountability
I don't think crews have a great understanding of PEA they don't necessarily know or fully understand the potential for reversible causes". (SP2)
Clinical leadership and moral judgements
"I would say that from my experience of it that you can make a decision with a degree of separation from what's going on so you can take a reasoned history and then consider the factors without the background noise if you like and the human factors that come into play from a stressful on scene environment". (SP4)

Supportive tools for cessation of resuscitation decisions

The 'checklist' as "I think the fallback option which is used is just follow a checklist in order for safe practice". (SP1) a safety net

Checklist deviation and sound clinical reasoning

Checklist and moving forward "cessation of resuscitation checklist which we should use which helps govern our decision making......if a patient doesn't fall within that then we have to be pretty confident and be very careful about calling a cessation of resuscitation attempt". (SP1)
"I do think the checklist that we are using is almost double negative in terms of the questioning and it's still a little ambiguous in some areas". (SP3) 
Theme 1. Defining a futile resuscitation

Senior paramedics applied a multifactorial approach in order to identify a futile resuscitation and subsequent cessation. Advanced age, co-morbidities, persistent PEA, low $\mathrm{ETCO}_{2}$ and prolonged resuscitation duration were considered alongside a perceived poor life quality or the possibility of a natural end of life. All were balanced to provide a clinical view of the patient, enabling senior paramedics to apply their knowledge, experience and acumen to determine the resuscitation as futile:

"what is our threshold and why are we resuscing people who've just reached the end of their life - that's not a reversible event". (SP5)

Theme 2. The impact of ceasing resuscitation

Repeated exposure to ceasing resuscitation increased confidence and the decision-making process became more comfortable over time; however, senior paramedics highlighted that the decision to cease was not easy or clear:

"I got to a position of confidence sometimes I had to work at it I never felt it was a wrong decision to make or that I was second guessing myself once I've hung up but on occasion it took me a while to get there". (SP4)

Theme 3. Perceived conflict between senior and on-scene paramedics

Senior paramedics felt remote clinical decision-making provided a degree of separation from the scene of the cardiac arrest. At times senior paramedics felt conflict between themselves and the on-scene paramedic due to a perceived lack of

accountability and responsibility when making cessation of resuscitation decisions:

"I think that's an element of accountability paramedics in general aren't very comfortable with". (SP1)

Supportive tools cessation of resuscitation decisions

Senior paramedics highlighted that the PEA cessation checklist worked as a supportive decision tool. Deviation from the checklist was possible with careful considered clinical judgement. This introduced subjectivity between senior paramedics when ceasing resuscitation and as a consequence, variation to the decision-making process:

"we've got the checklist we can still step outside the check list... but I think if you've got all the SCA's in a room and ran some scenarios I'm not entirely convinced they'd make the same consistent decision". (SP5)

\section{Discussion}

This mixed methods study was employed to describe PEA patients when resuscitation was ceased, the association between variables known to prognosticate survival and the autonomous experience of senior paramedics making cessation of resuscitation decisions. Synthesis of the quantitative and qualitative data summarised three categories 1) PEA within a local context, 2) senior paramedic experience of ceasing resuscitation 3) multifactorial decision-making.

\section{PEA within a local context}

In this study support from senior paramedics was obtained in only $6 \%$ of PEA patients who died at home: Patients may have transitioned from PEA to asystole and managed according to existing termination of resuscitation criteria, clinical advice was sought elsewhere or the on-scene paramedic made an autonomous decision to cease resuscitation. Paramedic-led decisions for cardiac arrest was previously explored in a qualitative study which found personal beliefs and values influential, (14) however in this study, on-scene paramedic cessation of resuscitation decisions were not explored and therefore require further investigation. Previous studies found paramedics felt training to identify those patients with a chance of survival or when recovery is beyond all hope (futile resuscitation), inadequate. ${ }^{8}$ This view was supported by the senior paramedics, who highlighted the need for improved paramedic education, specific to PEA and resuscitation decisions. This finding may explain why some conflict was encountered between the senior and on-scene paramedic as the clinical reasoning as to why the resuscitation was ceased was different. This finding is similar to that of previous studies which found conflict between staff groups due to personal beliefs, moral practice, pressure and expectation. $(8,16)$ 
Senior paramedics highlighted the challenges of remote clinical support. The on-scene paramedic was required to communicate patient information by telephone, adding to the pressures and time critical nature of resuscitation. The on-scene paramedic was able to build a patient history, including the environmental context, enabling a visual interpretation of the patients physical condition, including frailty. That said, the benefits of remote clinical support were considered by the National Institute of Health Care Excellence, finding that unnecessary conveyence, specifically in remote areas could be reduced. (17) The benefits of remote clinical support in this study found that senior paramedics had time to balance the benefit versus harm of continuing or ceasing resuscitation, which in turn reduced human factors and bias.

\section{Paramedic experiences of ceasing resuscitation}

In this study, remote clinical decisions were supported using a locally derived checklist. Deviation from the checklist occurred when senior paramedics applied clinical judgement to cease the resuscitation, as patient recovery was felt beyond hope. Checklists to support clinical decisions in a previous study were found

insufficient, as they omitted the multiple factors were required to build a holistic view of the patient.(19) This finding was congruent with the results of this study, as senior paramedics balanced multiple factors using clinical judgement, a vital process when deciding to continue or cease resuscitation.

In total senior paramedics documented 161 different rationales for ceasing resuscitation with multiple, yet different rationales for each patient. This result suggests senior paramedics introduced an element of subjectivity to the decision-making process. The decision to cease resuscitation, informed by their clinical judgement was found to be underpinned by their knowledge, experience and acumen, similar to that of a previous study.(9) Within the literature, this variability in practice was found to be acceptable, as resuscitation decisions were found to be unique to the circumstances of the cardiac arrest. (20)

Senior paramedics felt patient survival was more likely when the cardiac arrest was witnessed, with bystander BLS, progression to a shockable rhythm, defibrillation and ROSC, similar to factors which prognosticate in the chain of survival. Witnessed cardiac arrest and bystander BLS were statistically significant, similar to an observational study which found the recognition of cardiac arrest positively associated with bystander BLS.(10) Whereas cessation of resuscitation in a natural end of life, co-morbidities, advanced age, poor life quality, prolonged no flow time, no bystander resuscitation and persistant PEA, were more comfortable decisions as the patient had no realistic hope of survival.

Senior paramedics interpreted a number of clinical features in which to prognosticate the patients chance of survival. Duration of resuscitation was key, albeit not a statistically significant result. The majority of senior paramedics felt after 20-40 mins of ALS resuscitation, if signs of life were absent, the patient was beyond survival. Previous research was unable to identified an optimal duration of resuscitation for PEA, reporting anywhere between 10 to 47 mins. $(21,22)$ In this study the mean ALS duration $(54$ minutes) was longer when compared to senior paramedic views and no assocation with defibrillation and ROSC was found. The average ETCO ${ }_{2}$ in this study was 2.3kPa. Senior paramedics did not base cessation decisions on $\mathrm{ETCO}_{2}$ alone, however, a persistant low value was interpreted as a poor indicator. A systematic review applied $\mathrm{ETCO}_{2}$ to prognosticate resuscitation futility and whilst associations between survival were found, a specific cut off value could not be determined. ${ }^{23}$ A slow heart rate with a wide QRS morphology, found in $92 \%$ of patients in this study was considered a terminal rhythm. These results were similar to one study which found a higher death rate in patients with a low, wide QRS, (24) however, incongruent with another study which reported ECGs as a prognostic marker inaccurate.(25) In this study, 44\% of patients progressed to a shockable rhythm and $16 \%$ achieved ROSC, albeit intermittent. Previous studies found progression to a shockable rhythm $(26,27)$ and ROSC $(6,7)$ increased the chance of survivability. This result suggests senior paramedics ceased resuscitation in patients with known factors which prognosticate survival.

This finding requires further investigation as there may be a difference between patients presenting with known factor from the chain of survival, where resuscitation was not successful, compared to those patients beyond all hope of survival, known as futile. In addition, investigating a value or threshold of the clinical features would be helpful to determine the difference between those patients where resuscitation should be continued and in those patients where resuscitation should be ceased.

\section{Multifactorial decision-making}

In this study, the multifactorial nature of decision-making was not exclusive, similar to that of other studies. (15) No association between patient characteristics or system factors were found, however, senior paramedics felt the interpretation and balance of each was vital to the decision-making process. This approach was important for patients with a perceived poor quality of life, multiple co-morbidities or 
when establishing a natural end of life event. Senior paramedics acknowledged the subjectivity of interpreting the impact of comorbidities and life quality, however, if the chance of survival with a good functional recovery was low, the resuscitation was ceased. In a previous study, co-morbidities were found to negatively impact on patient survival.(13,28,29) Arguably, co-morbidities do not infer a poor quality of life, however, assessing this remotely, whilst providing time critical care was challenging. Senior paramedics did identify advanced age, frailty, terminal disease, informed by family wishes as a natural end of life. As this is not a reversible cause of cardiac arrest the decision to cease resuscitation was made with confidence. Senior paramedics were well supported within the literature to interpret these factors to enable a balanced decision. $(28,29,30)$

Previously only clinical features have been investigated to prognosticate the outcome of resuscitation. $(4,13)$ This approach was not supported by the senior paramedics. The clinical features previously described were interpreted, however, they were balanced along with no flow time, advanced age, co-morbidities, duration of resuscitation and quality of life. This multifactorial approach was felt necessary, to ensure a hollistic, best interest and individual decision, a view supported within the literature $(13,19)$

\section{Conclusion}

Senior paramedics interpreted and applied a multifactorial approach to ceasing resuscitation for patients in PEA. Patient characteristics, clinical features and system factors were balanced using clinical judgement, found to be vital to the decision-making process. This hollistic approach questions the the appropriateness of paramedics making cessation of resuscitation decisions based on clinical features alone. As prognostic factors for survival were present within the sample, further investigation is warranted to examine the difference between those patients unsuccesfully resuscitated and those patients identified as futile.

\section{Limitations}

The quantitative sample size was small due to the limited involvement of senior paramedics. This may have introduced a type II error, where results lacked the statistical power to detect a difference that may exist. No power calculation was applied. Missing data was identified for $\mathrm{ETCO}_{2}(12 \%)$ and the effectiveness of bystander resuscitation or by whom it was conducted was not measured. One of the senior paramedic interviews was conducted by email, with no opportunity to probe the interviewee's responses.

\section{Abbreviations}

$\begin{array}{ll}\text { ALS } & \text { Advanced life support } \\ \text { BLS } & \text { Basic life support } \\ \text { ECG } & \text { Electrocardiogram } \\ \text { ETCO }_{2} & \text { End tidal carbon dioxide } \\ \text { PEA } & \text { Pulseless electrical activity } \\ \text { ROSC } & \text { Return of spontaneous circulation } \\ \text { SCA } & \text { Senior clinical advisor }\end{array}$

\section{Declarations}

\section{Ethics}

Institutional ethics approval was provided by the South western Ambulance Service NHS foundation Trust (ref 17-018) and the University of Plymouth (reference 1718424). Retrospective consent was not required as patient data was fully anonymised. Participant written consent for senior paramedic was gained.

\section{Consent for publication}

All data is fully anonymised. Written consent of was gained for each participant, including for publication.

\section{Availability of data and materials}


An anonymised patient data set and anonymised participant transcripts are available from the author on request.

\section{Competing interests}

The authors declares no competing interests.

\section{Funding}

This study was undertaken from a dissertation as part of a clinical research masters programme at the University of Plymouth, funded by the National Institute of Health Research.

\section{Authors' contributions}

AC is the lead author and principal investor for this study. SB made significant contributions to the study design, data collection strategy and editing of this manuscript. RE supervised the collaboration of this manuscript, making significant contributions to the statistical analysis, results, findings and overall editing.

\section{Acknowledgements}

The authors would like to thank Professor Jonathan Benger for his valuable input and guidance as the educational supervisor for this study. Thank you to the paramedics who participated and collaborators.

\section{Collaborators}

J. Lynde and $\mathrm{H}$. Trebilcock extracted the quantitative data. M Hawley piloted interview questions. L. Tremayne and E. Freeman second coded the qualitative data.

\section{Disclaimer}

The views expressed in this publication are those of the author(s) and not necessarily those of the National Health Service, the NIHR, the Department of Health and Social Care or the authors' employers.

\section{Author information}

$\mathrm{AC}$ is a research paramedic working in the South West of England. AC undertook this original research as part of a masters in clinical research, within the NIHR clinical academic pathway. AC continues to work clinically as a paramedic and recently won NIHR funding for a pre-clinical academic fellowship to write a clinical doctorate fellowship application to continue research focused on paramedic led resuscitation for cardiac arrest patients with pulseless electrical activity.

\section{References}

1. Beun L, Yersin B, Osterwalder J, Carron PN. Pulseless electrical activity cardiac arrest: Time to amend the mnemonic "4H\&4T"? Swiss Med Wkly. 2015;145(July):1-6.

2. OHCAO Project Team. Out-of-Hospital Cardiac Arrest Outcomes Registry Epidemiology Report, 2017 English Ambulance Services [Internet]. 2017 [cited 2019 May 17]. Available from:

https://warwick.ac.uk/fac/sci/med/research/ctu/trials/ohcao/publications/2015_epidemiology/ohcao_annual_report_2017_final.pdf

3. Soar J, Deakin C, Lockey A, Nolan J, Perkins G. Adult advanced life support [Internet]. 2015 [cited 2018 May 11]. Available from: https://www.resus.org.uk/resuscitation-guidelines/adult-advanced-life-support/\#reversible

4. Inokuchi S, Masui Y, Miura K, Tsutsumi H, Takuma K, Atsushi I, et al. A New Rule for Terminating Resuscitation of Out-of-Hospital Cardiac Arrest Patients in Japan: A Prospective Study. J Emerg Med [Internet]. 2017 Sep 1 [cited 2018 May 17];53(3):345-52. Available from: https://www.sciencedirect.com/science/article/pii/S0736467917304717

5. Skrifvars, Vayrynen, Kuisma, Castren, Parr, Silfverstople, et al. Comparison of Helsinki and European Resuscitation Council "do not attempt to resuscitate" guidelines, and a termination of resuscitation clinical prediction rule for out-of-hospital cardiac arrest patients found in asystole or pulseless electrical activit. Resuscitation [Internet]. 2010 Jun [cited 2017 Oct 10];81(6):679-84. Available from: http://www.ncbi.nlm.nih.gov/pubmed/20381229 
6. House M, Jackson M, Dinning J, McMeekin P. Derivation of a Termination of Resuscitation Clinical Decision Rule in the UK - Journal of Emergency Medical Services. J Emerg Med [Internet]. 2017 [cited 2017 Nov 10]; Available from:

http://www.jems.com/articles/print/volume-42/issue-8/features/derivation-of-a-termination-of-resuscitation-clinical-decision-rule-inthe-uk.html

7. IMP Corp. IBM How to cite IBM SPSS Statistics or earlier versions of SPSS [Internet]. 2016 [cited 2019 Jun 25]. Available from: http://www-01.ibm.com/support/docview.wss?uid=swg21476197

8. Nixon V. Professional Practice in Paramedic, Emergency and Urgent Care [Internet]. Nixon V, editor. Professional Practice in Paramedic, Emergency and Urgent Care. Oxford, UK: John Wiley \& Sons, Ltd; 2013 [cited 2020 Apr 1]. Available from: http://doi.wiley.com/10.1002/9781119464389

9. Mason J, Nixon V. Clinical Decision Making. In: Professional Practice in Paramedic, Emergency and Urgent Care [Internet]. Oxford, UK: John Wiley \& Sons, Ltd; 2017. p. 50-76. Available from: http://doi.wiley.com/10.1002/9781119464389.ch3

10. Viereck S, Møller TP, Ersbøll AK, Bækgaard JS, Claesson A, Hollenberg J, et al. Recognising out-of-hospital cardiac arrest during emergency calls increases bystander cardiopulmonary resuscitation and survival. Resuscitation [Internet]. 2017 Jun 1 [cited 2019 Mar 3];115:141-7. Available from: https://www.sciencedirect.com/science/article/pii/S0300957217301570

11. Reynolds JC, Grunau BE, Rittenberger JC, Sawyer KN, Kurz MC, Callaway CW. Association Between Duration of Resuscitation and Favorable Outcome After Out-of-Hospital Cardiac Arrest. Circulation [Internet]. 2016 [cited 2019 Feb 9];134:2084-94. Available from: http://clinicaltrials.gov.

12. Touma O, Davies M. The prognostic value of end tidal carbon dioxide during cardiac arrest: A systematic review. Resuscitation [Internet]. 2013;84(11):1470-9. Available from: http://dx.doi.org/10.1016/j.resuscitation.2013.07.011

13. Drennan IR, Case E, Verbeek PR, Reynolds JC, Goldberger ZD, Jasti J, et al. A comparison of the universal TOR Guideline to the absence of prehospital ROSC and duration of resuscitation in predicting futility from out-of-hospital cardiac arrest. Resuscitation [Internet]. 2017 [cited 2018 May 21];111:96-102. Available from: http://www.ncbi.nlm.nih.gov/pubmed/27923115

14. House M, Jackson M, Dinning J, McMeekin P. Derivation of a Termination of Resuscitation Clinical Decision Rule in the UK - Journal of Emergency Medical Services [Internet]. 2017 [cited 2017 Sep 24]. Available from: http://www.jems.com/articles/print/volume42/issue-8/features/derivation-of-a-termination-of-resuscitation-clinical-decision-rule-in-the-uk.html

15. Field R, Fritz Z, Baker A, Grove A, Perkins GD. Systematic review of interventions to improve appropriate use and outcomes associated with do-not-attempt-cardiopulmonary-resuscitation decisions. Resuscitation [Internet]. 2014 Nov 1 [cited 2018 Nov 7];85(11):141831. Available from: https://www.sciencedirect.com/science/article/pii/S0300957214007266

16. Bossaert L, Perkins G, Askitopoulou H, Raffay V, Greif R, Haywood K, et al. European Resuscitation Council Guidelines for Resuscitation 2015 Section 11. The ethics of resuscitation and end-of-life decisions on behalf of The ethics of resuscitation and endof-life decisions section Collaborators 1. Resuscitation [Internet]. 2015 [cited 2018 Jun 26];95:302-11. Available from: http://dx.doi.org/10.1016/j.resuscitation.2015.07.033

17. Erbay H. Some Ethical Issues in Prehospital Emergency Medicine. Turkish J Emerg Med 2014;14:193-8. Available from: http://www.ncbi.nlm.nih.gov/pubmed/27437517. [Accessed 14 May 2019].

18. NICE. Chapter 4 Paramedic remote support Emergency and acute medical care in over 16s: service delivery and organisation NICE guideline. Emergency and acute medical care 2017. Available from: https://www.nice.org.uk/guidance/gidcgwave0734/documents/draft-guideline-4. [Accessed 19 Feb 2019].

19. Nqala M, Rout C, Aldous C. Remote clinical support by telephone for rural district hospital medical officers in the Eastern Cape. South African Fam Pract. 2015;57:286-90. Available from: https://www.tandfonline.com/doi/full/10.1080/20786190.2015.1055671. [Accessed 2 May 2019].

20. Mason J, Nixon V. Clinical Decision Making. Professional Practice in Paramedic, Emergency and Urgent Care. Oxford, UK: John Wiley \& Sons, Ltd; 2017. p. 50-76.

21. The British Medical Association, Resuscitation Council (UK) and the Royal College of Nursing. Decisions Relating to Cardiopulmonary Resuscitation: a joint statement from the British Medical Association, the Resuscitation Council (UK) and the Royal College of Nursing. J Med Ethics 2016;27:310 P - 316.

22. Andrew E, Nehme Z, Lijovic M, et al. Outcomes following out-of-hospital cardiac arrest with an initial cardiac rhythm of asystole or pulseless electrical activity in Victoria, Australia. Resuscitation. 2014;85:1633-9.

23. Reynolds J, Grunau B, Rittenberger J, et al. Association Between Duration of Resuscitation and Favorable Outcome After Out-ofHospital Cardiac Arrest. Circulation 2016;134:2084-94. Available from: http://clinicaltrials.gov. [Accessed 9 Feb 2019]. 
24. Touma O, Davies M. The prognostic value of end tidal carbon dioxide during cardiac arrest: A systematic review. Resuscitation 2013;84:1470-9. Available from: http://dx.doi.org/10.1016/j.resuscitation.2013.07.011

25. Skjeflo G, Nordseth T, Loennechen J, et al. ECG changes during resuscitation of patients with initial pulseless electrical activity are associated with return of spontaneous circulation. Resuscitation 2018;127:31-6. Available from: http://www.ncbi.nlm.nih.gov/pubmed/29621571. [Accessed 6 Feb 2019].

26. Ho M, Gatien M, Vaillancourt C, et al. Utility of prehospital electrocardiogram characteristics as prognostic markers in out-of-hospital pulseless electrical activity arrests. Emerg Med J 2018;35:89-95. Available from: http://www.ncbi.nlm.nih.gov/pubmed/29055890. [Accessed 23 Nov 2019].

27. Goto Y, Funada A, Goto Y. Relationship Between the Duration of Cardiopulmonary Resuscitation and Favourable Neurological Outcomes After Out-of-Hospital Cardiac Arrest. J Am Heart Assoc. 2016; 5:002819. Available from: http://jaha.ahajournals.org/lookup/doi/10.1161/JAHA.115.002819. [Accessed 11 Oct 2019].

28. Han K, Lee S, Lee E, et al. Prognostic Value of the Conversion to a Shockable Rhythm in Out-of-Hospital Cardiac Arrest Patients with Initial Non-Shockable Rhythm. J Clin Med 2019;8:644. Available from: https://www.mdpi.com/2077-0383/8/5/644. [Accessed 1 April 2020].

29. Field R, Fritz Z, Baker A, et al. Systematic review of interventions to improve appropriate use and outcomes associated with do not attempt cardiopulmonary resuscitation decisions. Resuscitation 2014;85:1418-31. Available from: https://www.sciencedirect.com/science/article/pii/S0300957214007266. [Accessed 7 Nov 2019].

30. Bossaert L, Perkins G, Askitopoulou H, et al. European Resuscitation Council Guidelines for Resuscitation 2015. Section 11. The ethics of resuscitation and end-of-life decisions. Resuscitation. 2015;95:302-11.

31. Ågård A, Herlitz J, Castrén M, et al. Guidance for ambulance personnel on decisions and situations related to out-of-hospital CPR. Resuscitation. 2012;83:27-31. 